\title{
Fibrin Glue in Interventional Radiology: How to Use It
}

\author{
Department of Diagnostic Radiology, Fukui-ken Saiseikai Hospital, Japan
}

Shiro Miyayama, Masashi Yamashiro, Rie Ikeda, Junichi Matsumoto, Nobuhiko Ogawa, Naoko Sakuagawa

\begin{abstract}
A fibrin glue kit consists of separate solutions of fibrinogen and thrombin that instantly coagulate when mixed together and can be used as an embolic agent and tissue adhesive in several interventional procedures, such as the closure of enterocutaneous, postoperative pancreatic, and bronchopulmonary fistulas, embosclerosis of biloma, and portal vein embolization. Separate and simultaneous injections of fibrinogen and thrombin solutions at the target site are necessary; therefore, insertion of two catheters or a balloon catheter with multiple lumen is required. The combined use of metallic coils is also effective for a large fistula as the coils can provide a matrix for retaining the fibrin glue, in addition to partially occluding the fistulous tract. Mixing contrast medium or iodized oil with a thrombin solution is also key to achieving radiopacity.
\end{abstract}

Key words: fibrin glue, embolic agent, tissue adhesive, interventional procedure

(Interventional Radiology 2021; 6: 122-129)

\section{Introduction}

Fibrin glue has been used since the 1970s in surgical procedures for hemostasis and fistula closure. It is a unique material that can coagulate at the desired site and promote wound healing [1, 2]; therefore, it has also been used in several vascular and non-vascular interventional procedures [3-10].

Two commercially available fibrin glue kits, Bolheal (Teijin, Tokyo, Japan) and Beriplast P Combi-set tissue adhesion (CSL Behring, Tokyo, Japan), as well as fibrinogen created from the autologous blood of the patient, are used in Japan. These products are equipped with dual-chamber applicator attachments of various lengths to ensure that coagulation occurs at the target site. However, the applicators are rigid and short; therefore, some techniques and devices are required to use the fibrin glue effectively in interventional procedures.

In this manuscript, we describe the use of the fibrin glue in interventional procedures for several clinical conditions.

\section{Characteristics of the Fibrin Glue}

The fibrin glue is a hemostatic or adhesive product made from human plasma and consists of two components: fibrinogen and thrombin. Thrombin acts as an enzyme and converts fibrinogen into fibrin in 10-60 seconds. The fibrin monomers aggregate together to form a coagulum, and thrombin-activated factor XIII (XIIIa) forms cross-links among different monomers acting as stabilizers. Calcium ions play a major role as co-factors in fibrin polymer formation and coagulum stability processes (Fig. 1)[1]. The fibrin glue can also stimulate penetration of the fibrin mass by fibroblasts and promote early adhesion between tissues via the generated fibrin mass. This series of actions can promote healing through the absorption of proteins, including fibronectin, and accelerate the production of collagen in the wound area [2]. The resultant coagulum is completely absorbed during wound healing without a foreign body reaction or extensive fibrosis [1]. Thus, the fibrin glue is a biocompatible and ideal material for several interventional procedures, although its cost is higher than that of other embolic agents. 


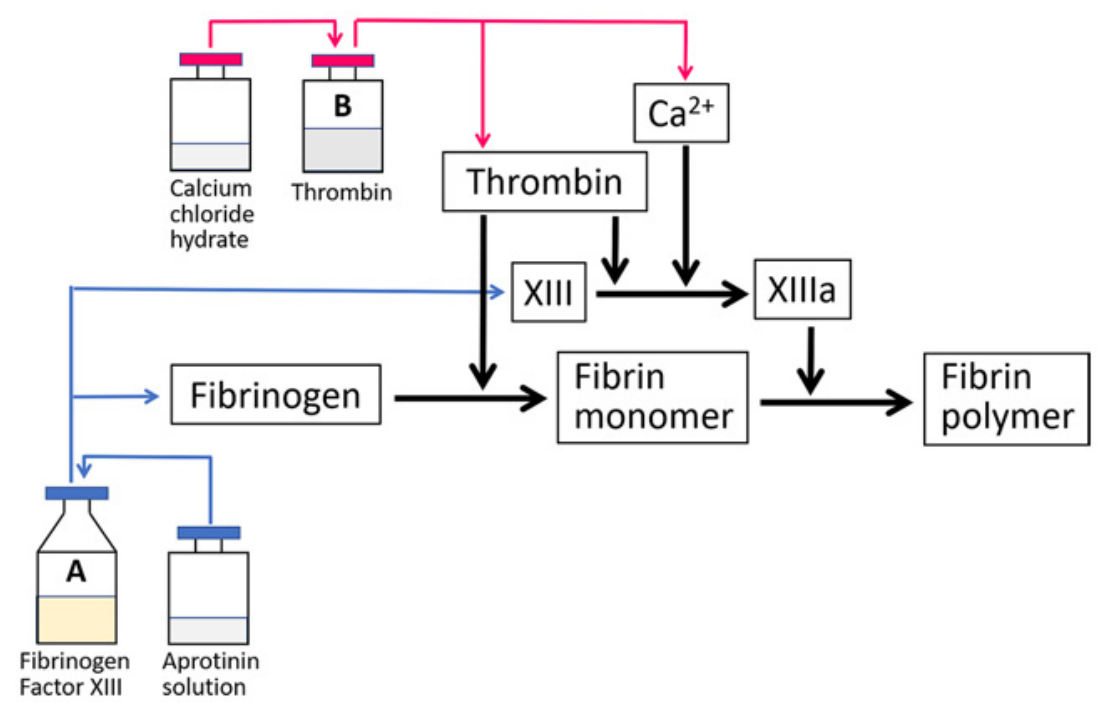

Fig. 1. The mechanism of action of fibrin glue

The thrombin solution has no viscosity; therefore, a small amount of contrast medium or iodized oil (Lipiodol 480, Guerbet Japan, Tokyo, Japan) can be mixed with it to achieve radiopacity, if necessary. On the contrary, the fibrinogen solution is viscous and difficult to mix with contrast or iodized oil.

\section{Fibrin Glue in Non-vascular Intervention}

The fibrin glue is mainly used for fistula closure. The procedure should be performed when the abscess cavity is shrunk, the fistula is fully mature, and local infection can be controlled.

\section{Closure of an enterocutaneous fistula (ECF)}

An ECF is an abnormal connection between the alimentary tract and skin created following abdominal surgery (the risk is as high as 85\%), inflammatory bowel disease (most commonly Crohn's disease), abscess, and ulcerating tumors. ECFs allow enteric contents to contaminate sterile spaces and may cause infection and sepsis, and the reported mortality rate from ECFs ranges from $10 \%$ to $30 \%$ [3].

Surgical fistula resection has recurrence rates of as high as $20 \%$ and mortality rates of as high as $7 \%$ [3]; therefore, interventional radiology can offer potential solutions for ECF closure or symptom reduction, except for cases with complications of underlying inflammatory bowel disease or malignancy, and cases requiring a repair of abdominal wall defects. In Western countries, several forms of plugs made from extracellular matrix (ECM) products are used to close ECFs [3]; however, there are no commercially available ECM plugs in Japan. Therefore, the fibrin glue or n-butyl-2cyanoacrylate (NBCA) is mainly used to close the ECF, with the fibrin glue being more suitable for this purpose as it causes fewer inflammatory reactions than NBCA.

The attached dual-chamber applicator cannot be used for fistula closure because of its short length. However, there are no standard techniques for ECF closure using the fibrin glue. Our technique is as follows: After sterilization of the skin, two straight or angled-tipped 4-F catheters are advanced into the fistulous tract as distally as possible along a 0.035-inch hydrophilic guidewire under fluoroscopic guidance. Thereafter, $0.5 \mathrm{~mL}$ of fibrinogen and thrombin solutions are slowly injected simultaneously through each catheter. One minute later, both the catheters are pulled back by approximately $5 \mathrm{~mm}$, and the injections of both the solutions are repeated. The procedure is continued until the entire length of the tract is sealed (Fig. 2). When the fibrin glue overflows outside the tract, both the catheters can be removed. If the ECF re-opens after the procedure, the fibrin glue injection should be repeated (Fig. 2).

\section{Closure of a postoperative pancreatic fistula (PPF)}

Pancreaticoduodenectomy is an operative method associated with many postoperative complications compared with other surgical procedures in the abdomen. In particular, a PPF may develop and lead to prolonged postoperative hospital stay or in-hospital death [4]. Most PPFs can be healed by percutaneous drainage alone; however, drain removal is impossible when the pancreatic fluid is continuously discharged through the catheter. Fistula closure with the fibrin glue is indicated for PPFs refractory to drainage. However, this method cannot be performed in cases where the alimentary canal side is completely closed or the pancreaticojejunostomy is completely separated [4].

The technique of closing the PPF is the same as that of ECF closure (Fig. 3). In a report by Okamoto et al. [4], all six PPFs could be healed by one fibrin glue injection; however, additional injections should be performed if it reopens after the procedure [5]. In a large PPF, the usefulness of the combination of metallic coils and fibrin glue has also been reported [5], as the metallic coils are considered to assist with fibrin glue retention in the fistula by providing a matrix, in addition to partially occluding the fistulous tract. 

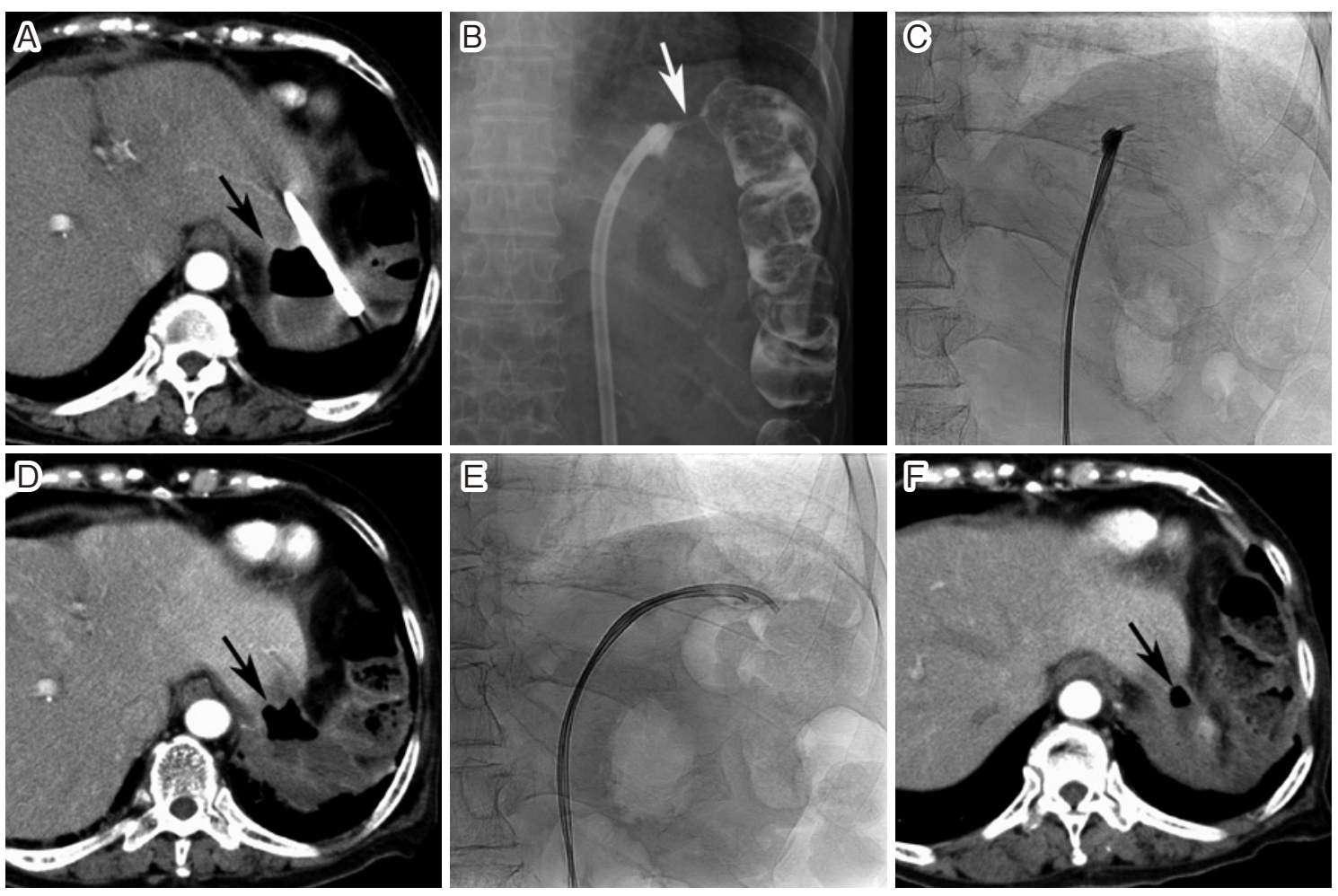

Fig. 2. Closure of an enterocutaneous fistula

A. The patient underwent a partial gastrectomy, debridement, and omentopexy for a perforated gastric ulcer. The discharge continued through the drain for 3 months and computed tomography (CT) showed a large cystic lesion containing air (arrow) near the drain and transverse colon. B. Fistulography revealed the splenic flexure of the transverse colon (arrow). C. A fibrin glue injection was performed using two 4-F catheters. D. However, the fistula reopened 8 days after the procedure. CT showed that the cystic lesion was still present (arrow). E. The drainage catheter was re-inserted, and the fibrin glue injection was repeated 15 days later. At that time, two 4-F catheters were advanced into the transverse colon through the fistula and the fibrin glue injection was continued while pulling back the catheters to seal the whole fistulous tract. F. The fistula healed after the second fibrin glue injection. CT performed 3 days after the procedure showed that the cystic lesion had markedly decreased in size (arrow).

\section{Closure of a bronchopulmonary fistula (BPF)}

Alveolar air leaks and BPFs are the most common complications after lung resection and significantly contribute to morbidity and mortality following a thoracic surgery. The fibrin glue is commonly used to treat air leaks during lung resection; however, persistent air leakage (longer than 5-7 days) occurs in up to $15 \%$ of patients and often requires additional treatments, such as repositioning of a pleural drainage catheter, chemical pleurodesis, and surgical repair, as well as closure of the BPF [6].

Intrabronchial and/or intrapleural injection of the fibrin glue is an effective treatment to close postoperative BPFs [7]. An intrabronchial injection is usually performed under bronchoscopy through a forceps channel, and a small amount of each solution is in turn injected through each catheter. The intrapleural injection is performed through the chest tube route or via direct puncture with two needles. In the case of a large BPF, a metallic coil placement before the fibrin glue injection is effective, and it can also be per- formed using a 4-F catheter via the forceps channel of the bronchoscope (Fig. 4).

If the intrapleural injection of fibrin glue is performed under computed tomography (CT) guidance, the contrast medium is mixed with a thrombin solution in a 1:1-3 ratio to render the mixture radiopaque on CT (Fig. 4).

\section{Embosclerosis of biloma}

Intrahepatic biloma formation is a severe complication of transarterial chemoembolization (TACE) for hepatocellular carcinoma. The intrahepatic bile ducts do not have a dual blood supply and are fed exclusively from the hepatic arterial branches, called the peribiliary vascular plexus (PBP). A possible mechanism of biloma formation after TACE is the development of peripheral bile duct necrosis with bile leakage and proximal bile duct stricture caused by microvascular damage to the PBP. In addition, biloma occurs more frequently after TACE of the normal liver as the PBP is dilated in the cirrhotic liver and can act as a portoarterial shunt to compensate for the decreased arterial flow to the bile duct 

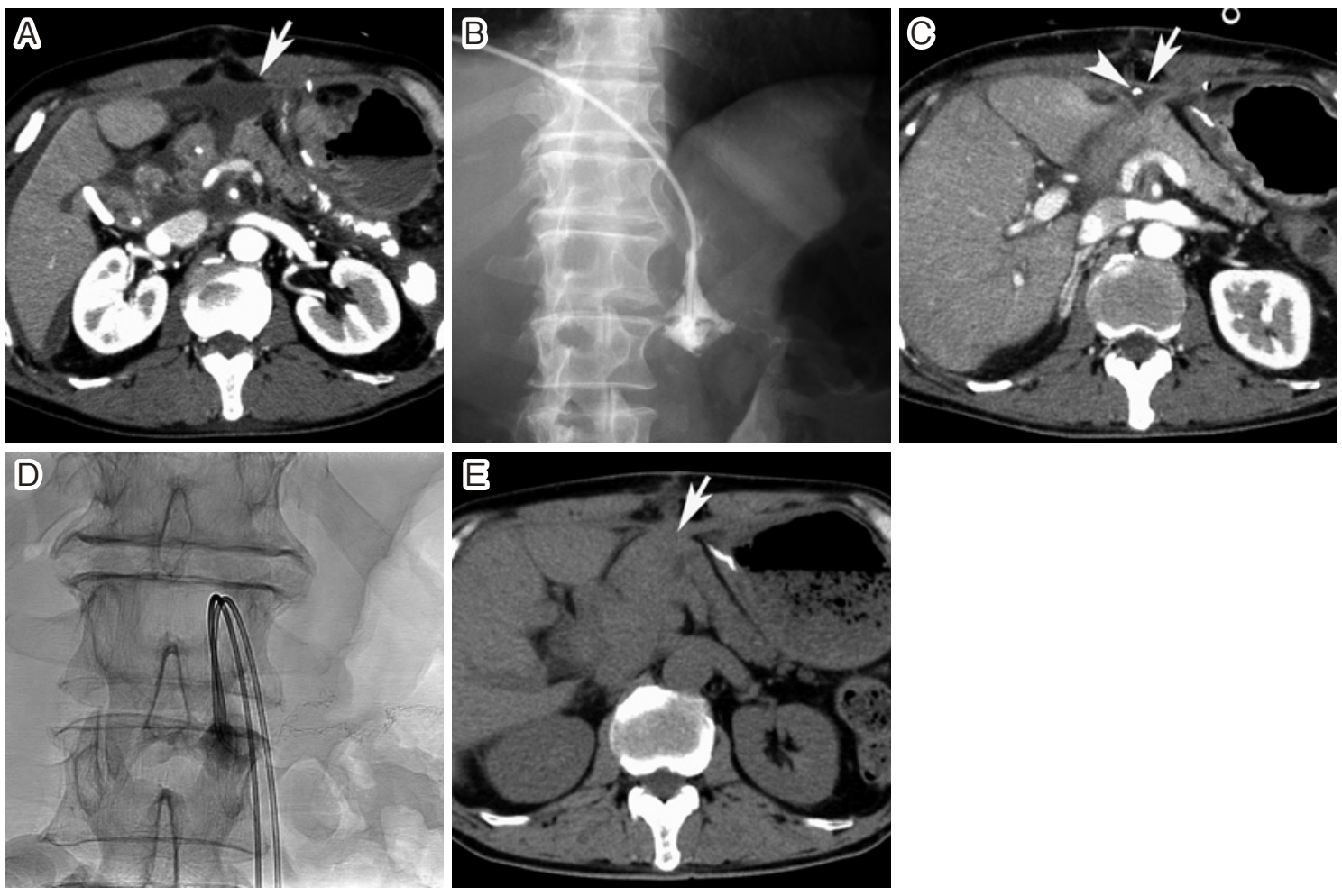

Fig. 3. Closure of a postoperative pancreatic fistula

A. Enhanced computed tomography (CT) performed 9 days after pancreaticoduodenectomy showed fluid collection at the pancreatojejunal anastomosis (arrow). B. Percutaneous drainage was performed, but pancreatic juice was continuously discharged through the catheter, although fistulography showed no opacification of the pancreatic duct. $C$. The discharge continued and enhanced CT performed 43 days after surgery showed a small amount of fluid at the anastomosis site (arrow). The arrowhead indicates the drainage catheter. D. On day 50 after surgery, a fibrin glue was injected into the fistula using two 4-F catheters. The fistula was closed after the procedure. E. Unenhanced CT performed 1 month after the procedure showed granulation at the anastomosis site (arrow).

[8]. Biloma also develops after percutaneous local ablation therapy and surgical resection of liver tumors. A small biloma is asymptomatic and requires no treatment, while a large and growing or infected biloma requires percutaneous transhepatic drainage. However, removal of the drainage catheter is impossible when bile discharge continues through the catheter. Although there are no standard treatments for biloma refractory to percutaneous drainage, the usefulness of embosclerosis of biloma using the fibrin glue has been reported [9].

Before performing embosclerosis of the biloma, it is necessary to treat the coexisting biliary stricture with placement of a plastic tube endoprosthesis. The procedure is almost the same as that of ECF and PPF closure; however, multiple sessions of fibrin glue injection are usually required [9]. Therefore, the access route to the biloma should be maintained by a drainage catheter until bile leakage is stopped. Our technique of biloma embosclerosis is as follows: a 0.035 -inch guidewire is advanced into the biloma through the drainage catheter, and the catheter is exchanged to an 8$\mathrm{F}$ sheath. After insertion of three 0.035 -inch guidewires into the biloma cavity through the sheath, the sheath is withdrawn. Thereafter, two 4-F angiography catheters and a new drainage catheter are advanced into the biloma cavity side by side over the wire, and stepwise, separate injections of fibrinogen and thrombin solutions $(0.5-1 \mathrm{~mL}$ at $1 \mathrm{~min}$ intervals) are performed through each angiography catheter (Fig. 5). After the fibrin glue injection, the two angiography catheters are withdrawn, and the drainage catheter is left in place. The procedure is repeated until the bile discharge is stopped.

\section{Fibrin glue in Vascular Intervention}

The fibrin glue has also been used in portal venous interventions [10], although it is an off-label use.

\section{Preoperative portal vein embolization (PVE)}

PVE has become a widely accepted method for inducing hypertrophy of the future liver remnant to prevent hepatic failure related to extensive hepatic resection. Many embolic materials, such as absolute alcohol, NBCA, gelatin sponge, coils, vascular plugs, microspheres, and fibrin glue, have been used for PVE.

The percutaneous transhepatic approach is most commonly used worldwide to perform PVE. Two access routes 

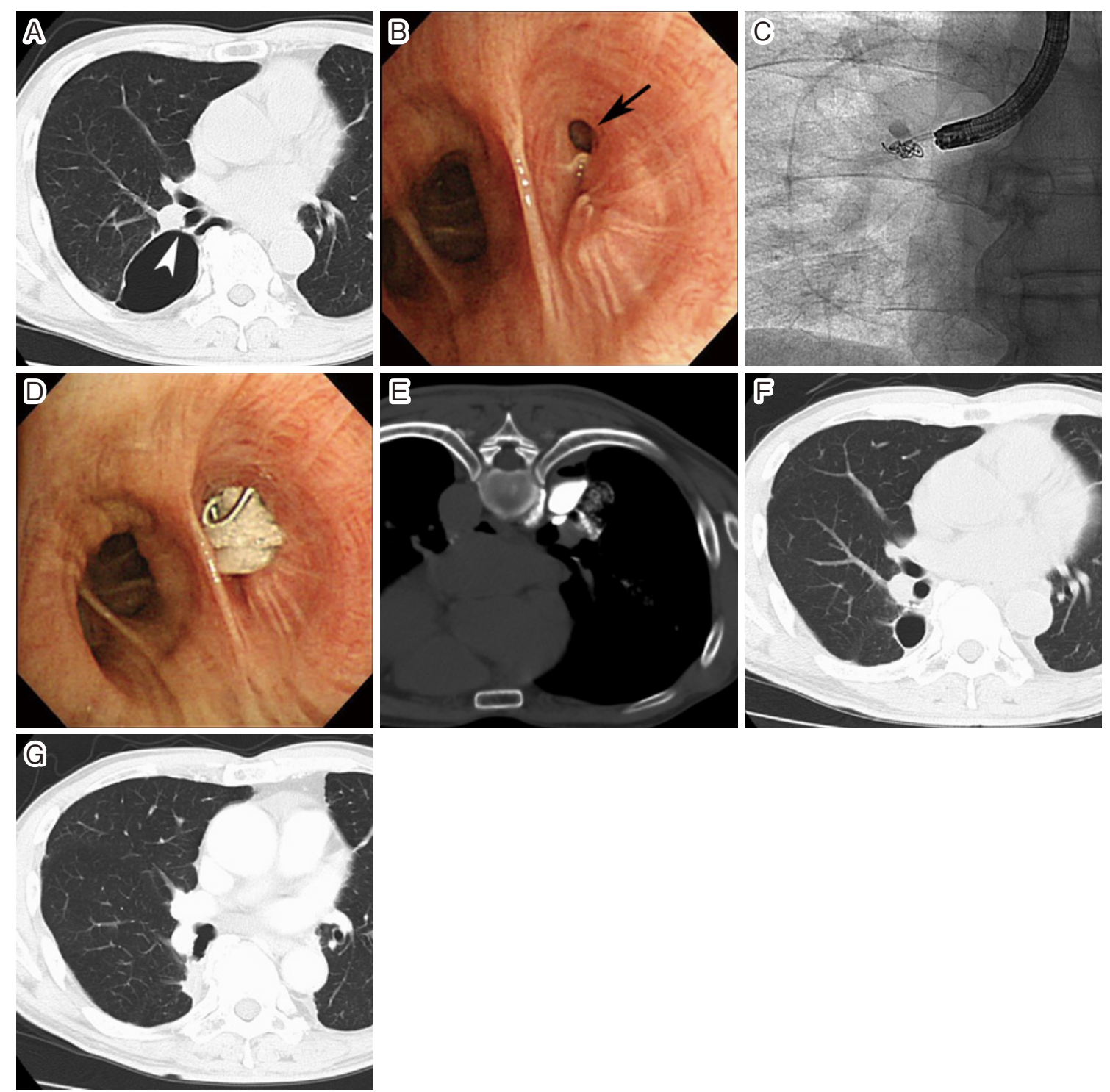

Fig. 4. Closure of a postoperative bronchopleural fistula

A. Computed tomography (CT) performed 1 year after partial resection of the right lower lobe showed postoperative bronchopleural fistula (BPF) (arrowhead) and localized pneumothorax. Intrabronchial fibrin glue injection into BPF was repeated twice, and both the intrabronchial and intrapleural fibrin glue injections were also performed once, but the fistula was not closed. B. Fourth attempt of BPF closure was performed. Bronchoscopy showed a large BPF (arrow). C. Two metallic coils were deployed into the fistula through a 4-F catheter. D. Thereafter, a fibrin glue injection was performed under bronchoscopy. E. Fibrin glue mixed with contrast medium was also injected into the pleural cavity under CT guidance. F. CT performed 3 months after the procedure showed that BPF was successfully occluded and the cavity of localized pneumothorax had markedly decreased in size. G. CT performed 2 years after the procedure showed the disappearance of the localized pneumothorax.

can be selected: the ipsilateral and contralateral approaches, with the ipsilateral approach considered safer than the contralateral approach as the risk of damaging the future liver remnant can be reduced [10]. First, the portal vein is punctured under ultrasonography guidance, and a vascular sheath is introduced into the portal vein. The sheath size is changed according to the approach route and type of balloon catheter. A 5.5-F triple-lumen occlusion balloon (Selecon MP catheter II [CB-Y67STTB], Terumo Tokyo, Japan), which is used in the contralateral approach, requires a 7-F sheath, and a four-lumen double-occlusion balloon catheter (Selecon MP catheter II [CB-Y67SSB4]), which is used in the ipsilateral approach, requires an 8-F sheath. When a four-lumen double-balloon catheter is used, the proximal balloon is removed by a surgical scalpel to create a second side hole for the injection of fibrinogen or thrombin solutions [10]. Prior to embolization, iodized oil is mixed with the thrombin solution at a ratio of $1: 2$ or 2:3 to achieve radiopacity. After 

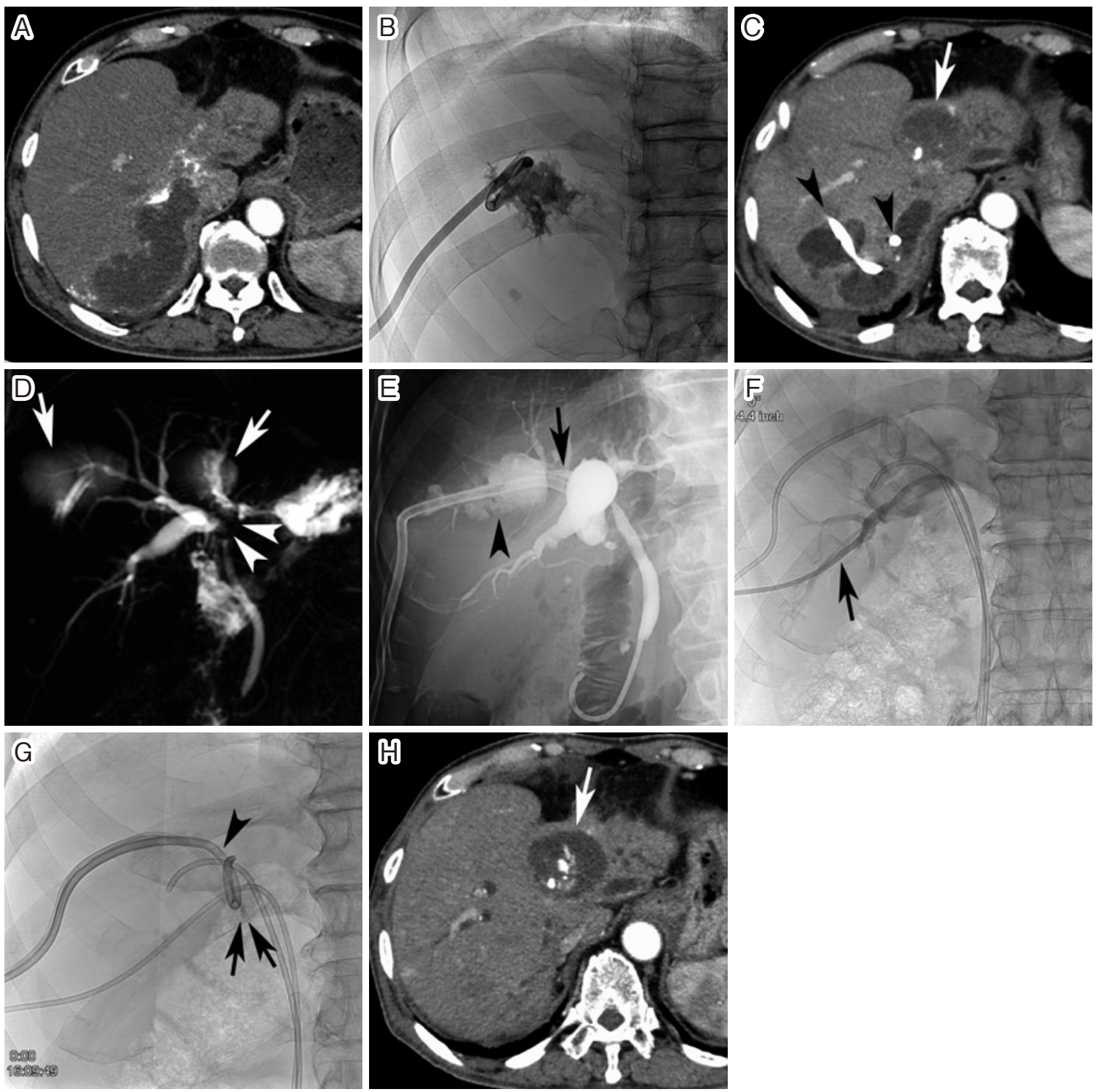

Fig. 5. Embosclerosis of intrahepatic biloma

A. Arterial-phase computed tomography (CT) performed 1 month after transarterial chemoembolization (TACE) showed a large biloma in the posterior segment of the right hepatic lobe. B. Percutaneous transhepatic biloma drainage was performed. $\mathrm{C}$. Bile discharge from the biloma continued despite multiple ablation sessions using 5\% ethanolamine oleate. CT performed 3 months after TACE showed the development of multiple bilomas in the right hepatic lobe. The arrowheads indicate the drainage catheter passing through three bilomas. The arrow also indicates a previously embolized tumor 9 years and 8 months ago. D. Magnetic resonance cholangiopancreatography showed bile duct strictures (arrowheads) at the hepatic hilum due to TACE, as well as the bilomas (arrows). E. A plastic endoprosthesis was endoscopically placed into the anterior segmental bile duct (arrow). However, the endoprosthesis could not be advanced into the posterior segmental bile duct branch. The biloma was also demonstrated (arrowhead). F. A drainage catheter was percutaneously advanced into the posterior segmental bile duct branch (arrow). G. Thereafter, a fibrin glue was injected into the biloma. The arrows indicate two 4-F catheters advanced into the biloma cavity. The arrowhead also indicates the drainage catheter in the biloma. Four sessions of the fibrin glue injection were performed at 3-4-day intervals. Thereafter, bile discharge was stopped and the drainage catheter could be removed. $\mathrm{H}$. Arterial phase CT performed 4 months after embosclerosis of biloma showed the disappearance of all bilomas. The arrow indicates the previously embolized tumor.

advancement of the balloon catheter at the desired position, the balloon is inflated, and fibrinogen and thrombin solutions are separately injected through each catheter lumen un- der fluoroscopy until filling in the major portal branches (Fig. 6). The balloon is kept inflated for 5 min to allow sufficient time for the fibrin glue to solidify completely. The 

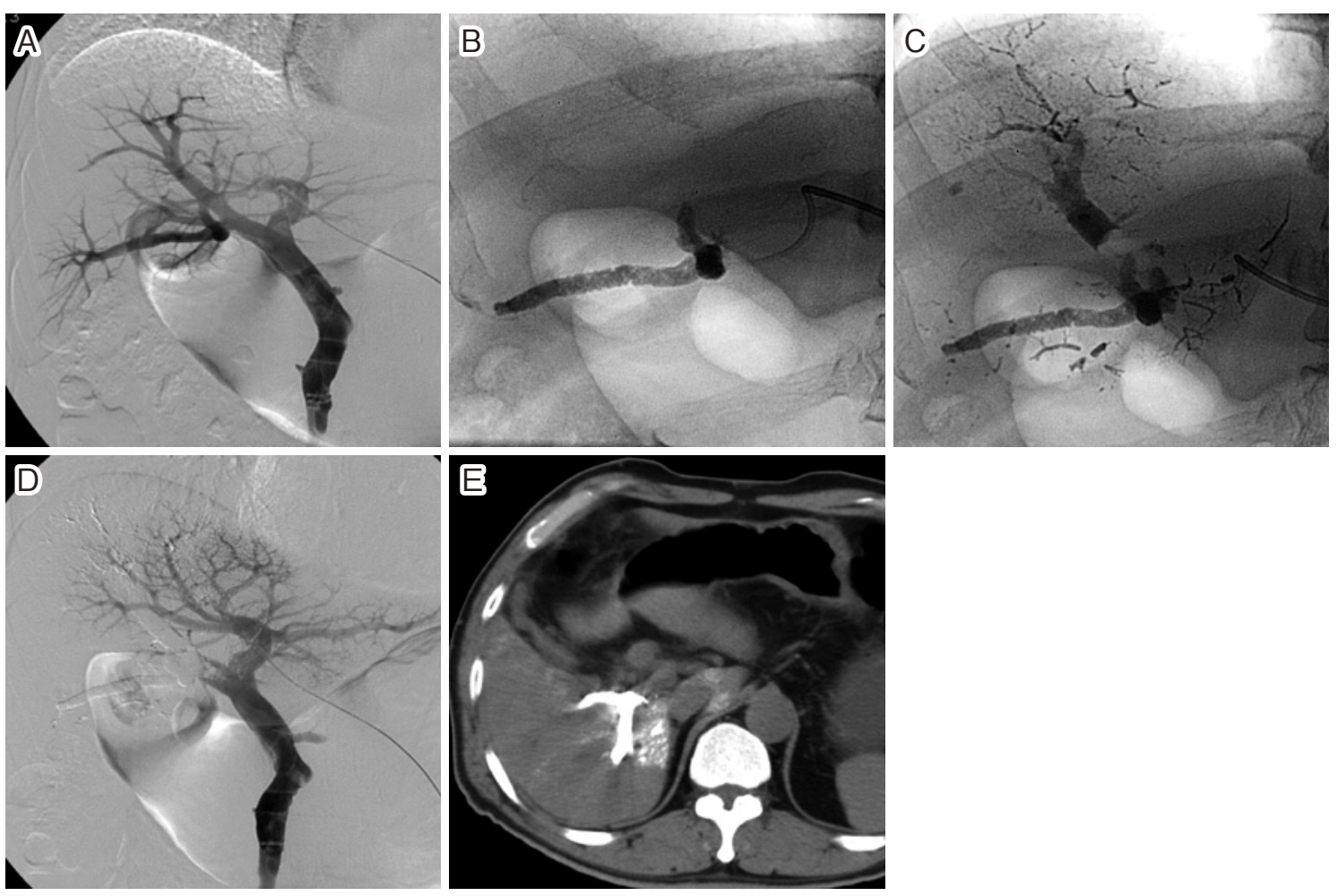

Fig. 6. Preoperative portal vein embolization for hilar cholangiocarcinoma

A. A 4-F pigtail catheter was advanced into the portal vein via the left portal branch and portography was performed. B. A balloon catheter was advanced into the posterior branch of the right portal vein and a fibrin glue mixed with iodized oil was injected under balloon occlusion. $C$. Thereafter, the fibrin glue was also injected into the anterior branch of the right portal vein under balloon occlusion. D. Portography performed immediately after portal vein embolization (PVE) showed complete occlusion of the right portal vein. E. Unenhanced CT performed 1 week after PVE showed the fibrin glues mixed with iodized oil in the right portal branches.

balloon is then deflated slightly so that the operator can confirm that no fibrin glue has refluxed into the portal vein trunk or non-targeted portal venous branch.

As mentioned above, the fibrin glue is expensive and a large-sized sheath is also required; therefore, it should be used in selected patients, such as those who cannot tolerate alcohol.

\section{Adverse effects}

Commercially available fibrin glue is made from human plasma, and fibrinogen created from autologous blood also requires thrombin from human or bovine plasma to form fibrin. Therefore, the fibrin glue may cause allergic reactions such as flushing, stinging, generalized urticaria, angioedema, bronchospasm, and anaphylaxis. Additionally, the risk of infections, such as those with human parvovirus B19, hepatitis viruses, and variant Creutzfeldt-Jakob disease, cannot be excluded. Therefore, it is necessary to inform patients of such risks before the procedure.

In fistula closure, aggravation of local infection may also develop after the fibrin glue injection; therefore, the procedure should be performed while controlling for local infection.

\section{Conclusion}

Fibrin glue is a unique and effective agent that can adhere to various fistulas and biloma cavities, and embolize the portal vein; therefore, radiologists should be familiar with the use of the fibrin glue.

Conflict of interest: The authors declare that they have no conflicts of interest and funding to report.

Disclaimer: Shiro Miyayama is one of the Senior Editors of Interventional Radiology and on the journal's Editorial Board. He was not involved in the editorial evaluation or decision to accept this article for publication at all.

\section{References}

1. Brennan M. Fibrin glue. Blood Rev. 1991; 5: 240-244.

2. Kubo M, Van de Water L, Plantefaber LC, et al. Fibrinogen and fibrin are anti-adhesive for keratinocytes: a mechanism for fibrin eschar slough during wound repair. J Invest Dermatol. 2001; 117: 1369-1381.

3. Rahman FN, Stavas JM. Interventional radiologic management and treatment of enterocutaneous fistulae. J Vasc Interv Radiol. 2015; 26: 7-19; quiz 20. 
4. Okamoto K, Koyama I, Hara K, et al. Fibrin glue closure for intractable pancreatic fistulae after pancreaticoduodenectomy. JOP. 2015; 16: 50-52.

5. Choi KM, Kim YD, Ahn JH. Closure of pancreatoduodenal fistula using vascular occluding coil embolization and fibrin glue injection: a case study. Korean J Hepatobiliary Pancreat Surg. 2013; 17: 75-78.

6. Malapert G, Hanna HA, Pages PB, Bernard A. Surgical sealant for the prevention of prolonged air leak after lung resection: metaanalysis. Ann Thorac Surg. 2010; 90: 1779-1785.

7. Fiorelli A, Odierna I, Scarano D, et al. Combined intrapleural and intrabronchial injection of fibrin glue for closing alveolar pleural fistula: a case report. J Cardiothorac Surg. 2019; 14: 171.

8. Sakamoto I, Iwanaga S, Nagaoki K, et al. Intrahepatic biloma formation (bile duct necrosis) after transcatheter arterial chemoem- bolization. AJR Am J Roentgenol. 2003; 181: 79-87.

9. Tanaka Y, Nakano A, Uchiyama J, et al. Effect of drainage and injection factor XIII with fibrinogen for hepatic biloma resulting from transcatheter arterial embolization in patients with hepatocellular carcinoma-Report of two cases-. Nisshoshi. 1998; 95: 238245 (in Japanese).

10. Gibo M, Unten S, Yogi A, et al. Percutaneous ipsilateral portal vein embolization using a modified four-lumen balloon catheter with fibrin glue: initial clinical experience. Radiat Med. 2007; 25 : 164-172.

Interventional Radiology is an Open Access journal distributed under the Creative Commons Attribution-NonCommercial 4.0 International License. To view the details of this license, please visit (https://creativecommons.org/licenses/by$\mathrm{nc} / 4.0 /)$. 